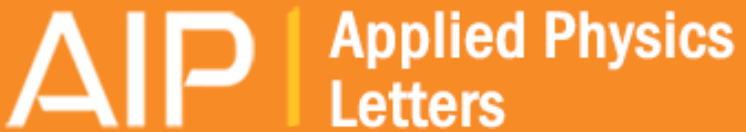

\section{(In,Ga)As/GaP electrical injection quantum dot laser}

M. Heidemann, S. Höfling, and M. Kamp

Citation: Applied Physics Letters 104, 011113 (2014); doi: 10.1063/1.4860982

View online: http://dx.doi.org/10.1063/1.4860982

View Table of Contents: http://scitation.aip.org/content/aip/journal/apl/104/1?ver=pdfcov

Published by the AIP Publishing

\section{Articles you may be interested in}

High performance InAs quantum dot lasers on silicon substrates by low temperature Pd-GaAs wafer bonding Appl. Phys. Lett. 107, 261107 (2015); 10.1063/1.4938205

High-gain and low-threshold InAs quantum-dot lasers on InP

Appl. Phys. Lett. 87, 243107 (2005); 10.1063/1.2146063

Long-wavelength laser based on self-assembled InAs quantum dots in InAlGaAs on InP (001)

Appl. Phys. Lett. 85, 1033 (2004); 10.1063/1.1779964

InAs quantum-dot GaAs-based lasers grown on AIGaAsSb metamorphic buffers

J. Appl. Phys. 94, 2133 (2003); 10.1063/1.1582229

The role of free carriers and excitons on the lasing characteristics of InAs/InGaAs quantum-dot lasers Appl. Phys. Lett. 82, 4812 (2003); 10.1063/1.1588377

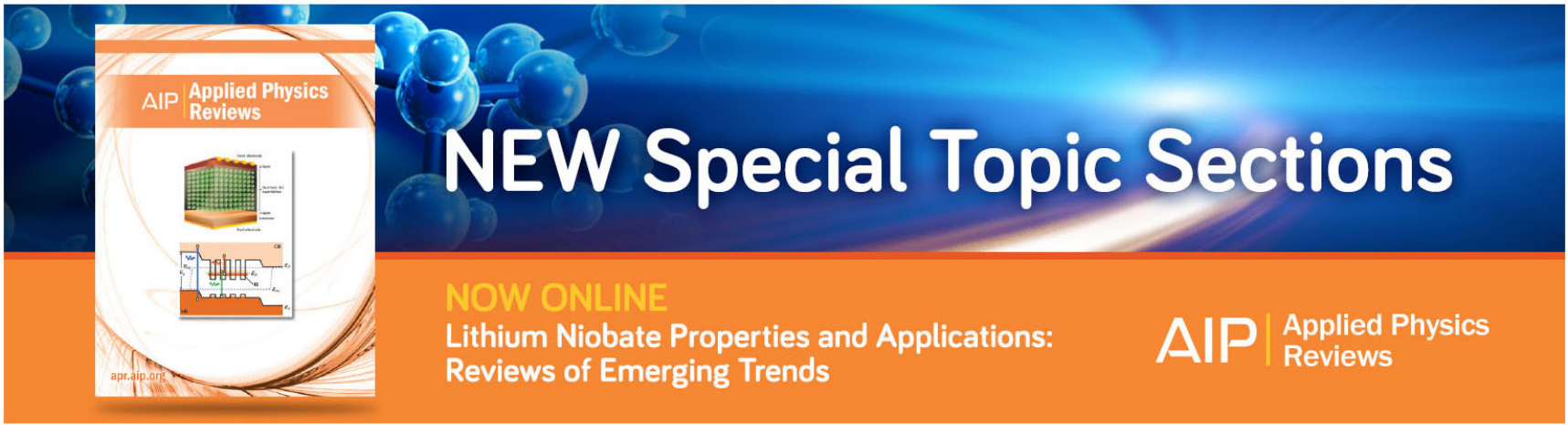




\title{
(In,Ga)As/GaP electrical injection quantum dot laser
}

\author{
M. Heidemann, ${ }^{\text {a) }}$ S. Höfling, and M. Kamp \\ Technische Physik, Universität Würzburg, Am Hubland, D-97074 Würzburg, Germany
}

(Received 11 July 2013; accepted 16 December 2013; published online 9 January 2014)

\begin{abstract}
The paper reports on the realization of multilayer (In,Ga)As/GaP quantum dot (QD) lasers grown by gas source molecular beam epitaxy. The QDs have been embedded in (Al,Ga)P/GaP waveguide structures. Laser operation at $710 \mathrm{~nm}$ is obtained for broad area laser devices with a threshold current density of $4.4 \mathrm{kA} / \mathrm{cm}^{2}$ at a heat-sink temperature of $80 \mathrm{~K}$. C 2014 AIP Publishing LLC.

[http://dx.doi.org/10.1063/1.4860982]
\end{abstract}

The monolithic integration of III/V optoelectronics with $\mathrm{Si}$ is of great interest for monolithic optoelectronic integrated circuits and optical interconnects. ${ }^{1}$ However, the epitaxy of well-established direct bandgap III/V compound semiconductors directly on $\mathrm{Si}$ is very difficult because of the large lattice mismatch, resulting in the formation of threading dislocations which prevent reliable laser operation. ${ }^{2}$ Among all binary III/V materials, GaP offers the lowest lattice mismatch to $\mathrm{Si}$ with only $0.37 \%$ at $300 \mathrm{~K}$, which ensures that a thin GaP film can be grown without significant degradation of the crystal quality. Complete lattice matching can be achieved with the dilute semiconductor $\mathrm{GaP}_{0.98} \mathrm{~N}_{0.02}$, but it is not suitable for the first nanometers of $\mathrm{Si}$ integration because of defect formation at the interface due to the creation of $\mathrm{Ga}$ vacancies by the addition of small concentrations of nitrogen. ${ }^{3}$ Since GaP has an indirect bandgap and therefore a poor radiative efficiency, ${ }^{4}$ the epitaxy of direct bandgap III/V materials on $\mathrm{GaP}$ is required, and a lot of different compounds have been investigated. ${ }^{5-12}$

The so far most successful material system on GaP is the dilute nitride $\mathrm{Ga}(\mathrm{NAsP})$ alloy. The strained $\mathrm{Ga}$ (NAsP) material system relies on a pseudo-direct bandgap character, since the nitrogen level is positioned below the conduction band even for an As-concentration of $100 \% .^{13}$ Laser operation up to room temperature has already been demonstrated $^{14-16}$ in devices with strained quantum wells based on this material system. In order to achieve a direct bandgap in nitrogen free compounds, (Ga,In)P and (Ga,In)As quantum dots (QDs) were fabricated under Stranski Krastanov growth conditions.

Although GaInP is a direct bandgap semiconductor for Gallium fractions under $71 \%,{ }^{17}$ an indirect type II strain induced transition takes place at the interface between the GaP barrier and the GaInP QDs, even for InP QDs, resulting in low photoluminescence (PL) intensities. ${ }^{5,6}$ The first results on (In, Ga)As QDs also showed low PL signals, ${ }^{18}$ indicating the same type of transition as for the $(\mathrm{Ga}, \mathrm{In}) \mathrm{P}$ QDs. A more detailed analysis of these QDs revealed a crossover from indirect to direct transitions for certain In concentrations and shapes of the QD. ${ }^{12}$

\footnotetext{
${ }^{\text {a)} E l e c t r o n i c ~ m a i l: ~ m a t t h i a s . h e i d e m a n n @ p h y s i k . u n i-w u e r z b u r g . d e . ~ T e l .: ~+49-~}$ 931-31-86256.
}

In this paper, we present results on the epitaxial growth of (In, Ga)As QDs on GaP (001) substrate, in particular, investigation of the size distribution and density of the QDs, as well as the optical properties under PL excitation. Finally, electrical injection lasing is demonstrated for (In,Ga)As/GaP QDs embedded in (Al,Ga)P/GaP-based waveguide structures.

The epitaxial growth of the samples was performed in an EIKO gas source molecular beam epitaxy (GSMBE) on (100) S:GaP wafers (n-doped). Group-III elements were provided by solid sources, while Group-V materials As and $\mathrm{P}$ were provided by the gases $\mathrm{AsH}_{3}$ and $\mathrm{PH}_{3}$, respectively. Due to the large bandgap, GaP-substrates are transparent in the infrared region, so the substrate surface temperature could not be measured with conventional pyrometry. Instead, it was determined via the temperature dependence of the bandgap by optical spectroscopy. ${ }^{19}$

A schematic of the laser structure is given in Fig. 1. The cladding layer and waveguide structure were optimized by solving a type of Helmholtz equation for an arbitrary onedimensional real refractive index profile with refractive index of 3.12, 3.25, and 3.84 for $\mathrm{Al}_{0.3} \mathrm{Ga}_{0.7} \mathrm{P}, \mathrm{GaP}$, and $\mathrm{In}_{0.3} \mathrm{Ga}_{0.7} \mathrm{As}$, respectively, at $710 \mathrm{~nm}^{20}$ The structure consists of a $300 \mathrm{~nm}$ n-type GaP buffer layer, followed by a $1.5-\mu \mathrm{m}$-thick n-type $\mathrm{Al}_{0.3} \mathrm{Ga}_{0.7} \mathrm{P}$ cladding layer, a $0.28-\mu \mathrm{m}$-thick undoped $\mathrm{GaP}$ waveguide with 3 layers of QDs separated by $10 \mathrm{~nm} \mathrm{GaP} \mathrm{bar-}$ riers placed at the center of the waveguide, a $1.5-\mu \mathrm{m}$-thick p-type $\mathrm{Al}_{0.3} \mathrm{Ga}_{0.7} \mathrm{P}$ upper cladding layer, and a $0.2-\mu \mathrm{m}$-thick $\mathrm{p}-\mathrm{GaP}$ contact layer. The large electron affinity of GaP makes the formation of good ohmic contacts to $\mathrm{p}-\mathrm{GaP}$ difficult. Therefore, a highly p-doped $10 \mathrm{~nm}$ graded $\mathrm{GaAs}_{\mathrm{x}} \mathrm{P}_{1-\mathrm{x}}$ layer was deposited on top of the structure in order to achieve good ohmic contacts with a measured contact resistivity of $1.1 \times 10^{-5} \Omega \mathrm{cm}^{2}$. Si and Be were used as $\mathrm{n}$ and $\mathrm{p}$ dopants and their concentrations in the structure determined by secondary ion mass spectroscopy. The n-doped and undoped regions were grown at a substrate temperature of $580^{\circ} \mathrm{C}$. The substrate temperature was lowered to $500^{\circ} \mathrm{C}$ for the growth of the $\mathrm{p}$-doped regions, since $\mathrm{Be}$ tends to segregate from the grown film. ${ }^{21}$ The growth rates of $\mathrm{Al}_{0.3} \mathrm{Ga}_{0.7} \mathrm{P}, \mathrm{GaP}$, and InGaAs were $1.5 \mu \mathrm{m} / \mathrm{h}, 1 \mu \mathrm{m} / \mathrm{h}$, and $0.3 \mu \mathrm{m} / \mathrm{h}$, respectively.

For the growth of the QD active region, the substrate temperature was reduced to $500^{\circ} \mathrm{C}$. The QDs were formed by the deposition of 3.5 monolayers of (In,Ga)As with a nominal indium composition of $40 \%$ on $10 \mathrm{~nm}$ of GaP, separated by $20 \mathrm{~s}$ growth interruption under As flux. The actual 


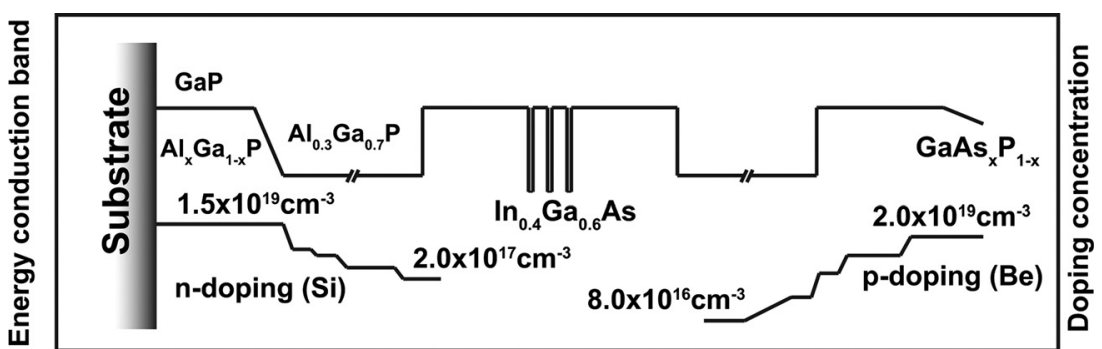

Growth direction

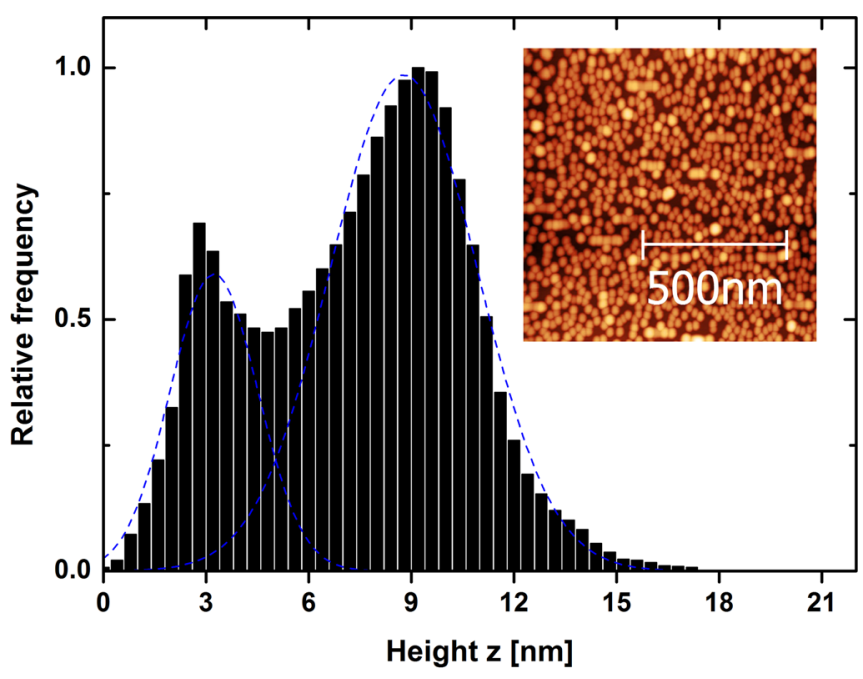

FIG. 2. Height distribution of 3.5 monolayers uncapped (In,Ga)As QDs measured with atomic force microscopy, grown at $500^{\circ} \mathrm{C}$ on $\mathrm{GaP}$ substrate with a high density of $8 \times 10^{10} \mathrm{~cm}^{-2}$.

In composition in these QDs is expected to be below 40\%, since In segregation from the QDs to the surrounding material reduces the In concentration inside the QDs. The driving force for this segregation is the strain acting at the growth surface, until the quantum dot structure is covered by a sufficiently thick capping layer. ${ }^{22}$ The optical and morphological properties of the QDs were analyzed with PL spectroscopy and atomic force microscopy (AFM) of QD samples grown under the same conditions.

Fig. 2 shows a $(1 \times 1) \mu \mathrm{m}^{2}$ AFM image of uncapped $(\mathrm{In}, \mathrm{Ga}) \mathrm{As} / \mathrm{GaP}$ QDs acquired in non-contact AFM mode, having a high QD density of $8 \times 10^{10} \mathrm{~cm}^{-2}$ and a bimodal height distribution with mean heights of $3.1 \pm 1.1 \mathrm{~nm}$ and $9.0 \pm 2.3 \mathrm{~nm}$. In order to study the band alignments of (In, Ga)As QDs embedded in GaP barriers, 6-band kp calculations together with Poisson- and strain equations are solved with parameters used from Ref. 23. Strain is taken into account by a continuum model on the basis of elasticity theory to calculate these strain fields. This is based on the Euler-Cauchy stress principle and Cauchy's fundamental postulate. Fig. 3 shows the calculated band alignment of (In, Ga)As QDs with geometry parameters acquired from the AFM measurement.

For the smaller QDs, the transition with the lowest energy is spatially direct, but indirect in k-space, taking place between the conduction band $X_{x y}$-point and the valence band $\Gamma$-point. For the larger QDs, the quantization energy of the level at the $\Gamma$-point is reduced, resulting in a transition with direct type-I nature. Because of the larger strain compared to the smaller QD, the lowest energy level of the X-point is at the interface between the QD and the $\mathrm{GaP}$ barrier at the $\mathrm{X}_{\mathrm{z}}$-point, similar to the $(\mathrm{Ga}, \mathrm{In}) \mathrm{P} / \mathrm{GaP}$ system. ${ }^{24}$ This is different compared to Ref. 25, where it was calculated that for small and large dots the lowest X-point is at $\mathrm{X}_{\mathrm{xy}}$, probably because the interface state was not taken into account. Despite the lowest X-point transition for larger dots, the results are in agreement with Ref. 25, where it was calculated that a ground transition of indirect type in reciprocal space occurs for smaller dots or low In content and a direct one for large QDs or high In content.

PL spectroscopy was performed by exciting the QD samples with a 405-nm continuous-wave laser diode. The (a)

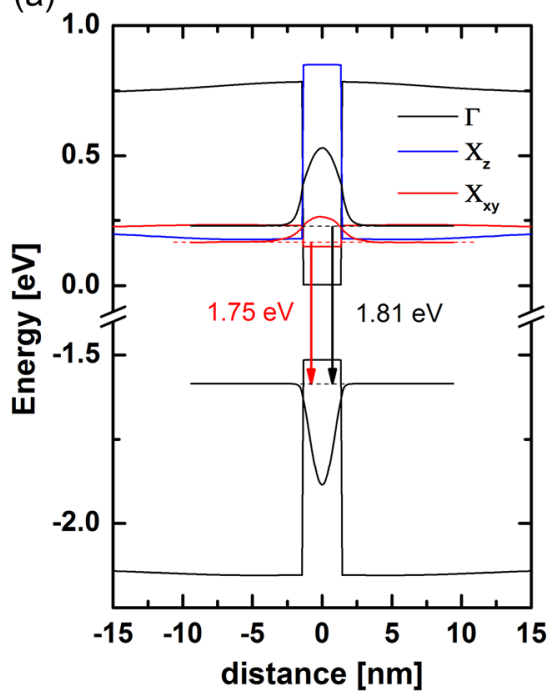

(b)

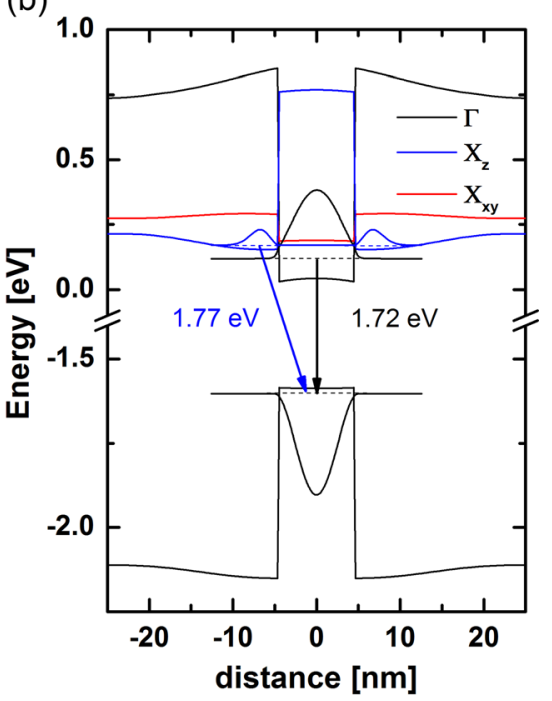

FIG. 3. 6-band $\mathrm{kp}$ calculation of the energy bands in a cylindrical $\mathrm{In}_{0.3} \mathrm{Ga}_{0.7} \mathrm{As} / \mathrm{GaP} \mathrm{QD}$ at $0 \mathrm{~K}$ with (a) $3.1 \mathrm{~nm}$ height and (b) $9.0 \mathrm{~nm}$ height and a diameter of $35 \mathrm{~nm}$. The energy of the bands is plotted along the central axis of the dot. 


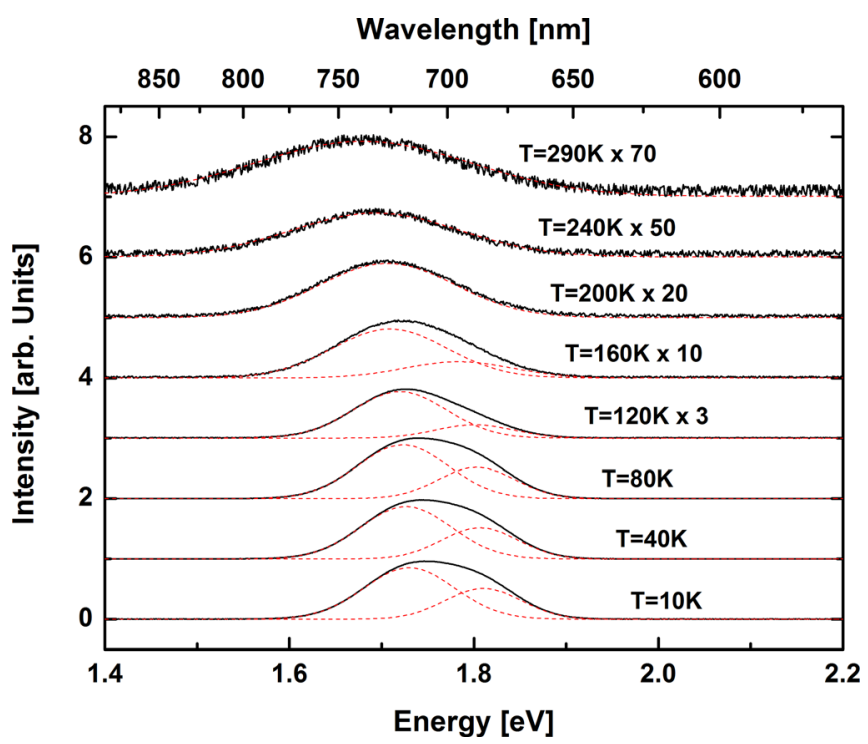

FIG. 4. Temperature dependent PL measurement of (In,Ga)As QDs. The dashed lines correspond to Gaussian fits of the two different QD size distributions.

power density was about $60 \mathrm{~W} / \mathrm{cm}^{2}$. The sample was cooled down in a helium closed-cycle cryostat to study PL from $10 \mathrm{~K}$ to room temperature. Fig. 4 presents the temperature dependent PL spectra of a single layer of (In, Ga)As/GaP QDs. At low temperatures, the emission of the QDs can be fitted by two Gaussian curves that correspond to the two different dot size distributions. The energies of the two peaks at $1.73 \mathrm{eV}$ and $1.82 \mathrm{eV}$ agree well with the simulated transitions energies of $1.72 \mathrm{eV}$ and $1.81 \mathrm{eV}$ obtained for a geometry based on AFM data. For increasing temperatures, the intensity of the smaller QDs decreases, until it vanishes at about $200 \mathrm{~K}$, which is attributed to the lower direct transition probability of these QDs, and only the larger QDs contribute to the PL signal.

For the characterization of laser layers, broad area devices have been processed by deposition of $50 \mu \mathrm{m}$ and $100 \mu \mathrm{m}$ wide $\mathrm{Ti} / \mathrm{Pt} / \mathrm{Au}$ metal stripes on the p-top contact and a $\mathrm{Ni} / \mathrm{AuGe} / \mathrm{Au}$-based substrate back contact. The samples were alloyed for $30 \mathrm{~s}$ at $400^{\circ} \mathrm{C}$ for ohmic contact formation. The finished laser bars were mounted p-side up in a variable temperature cryostat for temperature-dependent measurements under pulsed injection conditions.

Fig. 5 shows a low-temperature $(80 \mathrm{~K})$ output power characteristic from an as-cleaved, $50 \mu \mathrm{m}$ wide and $1 \mathrm{~mm}$ long QD laser. A clear threshold behavior of the output power is observed with a threshold current density of $4.2 \mathrm{kA} / \mathrm{cm}^{2}$. The inset of Fig. 5 shows an emission spectrum recorded at 2.15 A drive current, with the typical modal characteristic of a laser. At $140 \mathrm{~K}$, the threshold current density is $6.3 \mathrm{kA} / \mathrm{cm}^{2}$. Due to the current limit of $3 \mathrm{~A}$ in the experimental setup, laser operation could not be achieved at higher temperatures. For more homogenous QDs with a higher Indium content as well as an optimized laser design, a reduction of the threshold current density is expected, to achieve room temperature laser operation.

In conclusion, high density (In,Ga)As QDs $\left(8 \times 10^{10} \mathrm{~cm}^{-2}\right)$ were realized on GaP substrate. Band calculation showed that the indium content and size of the QDs

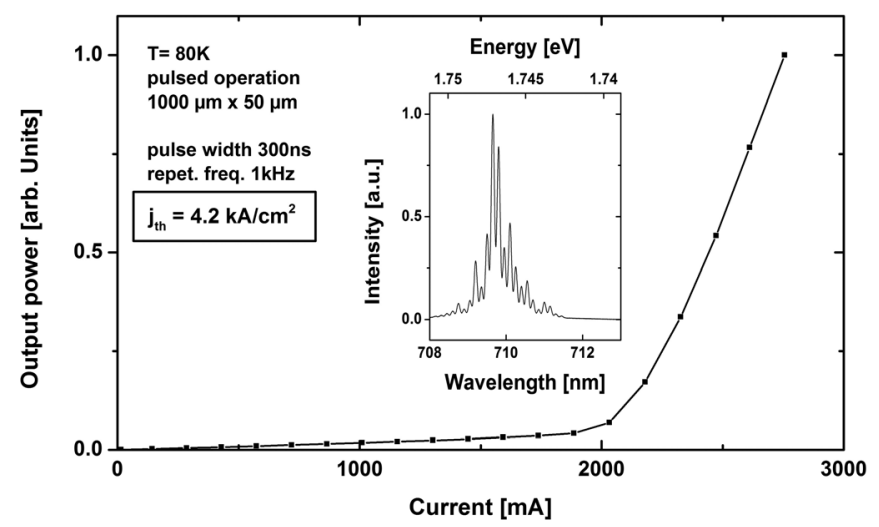

FIG. 5. Output power vs. current (L-I) characteristic at $80 \mathrm{~K}$. The inset shows the optical spectrum as a function of the emission wavelength which shows the expected modal characteristic of laser operation.

determine the type of transition. Temperature dependent photoluminescence analysis has verified this assumption. Based on these QDs broad area laser have been fabricated, showing laser activity in a nitrogen free device with a threshold current density of $4.2 \mathrm{kA} / \mathrm{cm}^{2}$ at $80 \mathrm{~K}$. Improvements in laser characteristics are expected by further optimization of the GSMBE growth conditions to optimize optical and electrical properties of the laser, so that this material system has a potential for applications integrating GaP based optoelectronics and Si-based microelectronics in the future.

Support given by M. Wagenbrenner is gratefully acknowledged.

${ }^{1}$ D. Liang and J. E. Bowers, Nat. Photonics 4, 511 (2010).

${ }^{2}$ S. F. Fang, K. Adomi, S. Iyer, H. Morkoç, H. Zabel, C. Choi, and N. Otsuka, J. Appl. Phys. 68, R31-R58 (1990).

${ }^{3}$ W. Li, M. Pessa, T. Ahlgren, and J. Decker, Appl. Phys. Lett. 79, 1094 (2001).

${ }^{4}$ M. R. Lorenz, G. D. Pettit, and R. C. Taylor, Phys. Rev. 171, 876 (1968).

${ }^{5}$ S. Gerhard, V. Baumann, S. Höfling, and A. Forchel, Nanotechnology 20, 434016 (2009).

${ }^{6}$ F. Hatami, V. Lordi, J. S. Harris, H. Kostial, and W. T. Masselink, J. Appl. Phys. 97, 096106 (2005).

${ }^{7}$ K. Umeno, Y. Furukawa, A. Wakahara, R. Noma, H. Okada, H. Yonezu, Y. Takagi, and H. Kan, J. Cryst. Growth 311, 1748 (2009).

${ }^{8}$ K. Umenon, Y. Furukawa, N. Urakami, R. Noma, S. Mitsuyoshi, A. Wakahara, and H. Yonezu, Physica E 42, 2772 (2010).

${ }^{9}$ Y. Song, P. J. Simmonds, and M. L. Lee, Appl. Phys. Lett. 97, 223110 (2010).

${ }^{10}$ T. S. Shamirzaev, D. S. Abramkin, A. K. Gutakovskii, and M. A. Putyato, Appl. Phys. Lett. 97, 023108 (2010).

${ }^{11}$ B. Kunert, K. Volz, J. Koch, and W. Stolz, Appl. Phys. Lett. 88, 182108 (2006).

${ }^{12}$ T. Nguyen Thanh, C. Robert, C. Cornet, M. Perrin, J. M. Jancu, N. Bertru, J. Even, N. Chevalier, H. Folliot, O. Durand, and A. Le Corre, Appl. Phys. Lett. 99, 143123 (2011).

${ }^{13}$ C. Robert, M. Perrin, C. Cornet, J. Even, and J. M. Jancu, Appl. Phys. Lett. 100, 111901 (2012).

${ }^{14}$ B. Kunert, S. Reinhard, J. Koch, M. Lampalzer, K. Volz, and W. Stolz, Phys. Status Solidi C 3(3), 614 (2006).

${ }^{15}$ B. Kunert, A. Klehr, S. Reinhard, K. Volz, and W. Stolz, Electron. Lett. 42(10), 601 (2006).

${ }^{16}$ N. Hossain, S. J. Sweeney, S. Rogowsky, R. Ostendorf, J. Wagner, S. Liebich, M. Zimprich, K. Volz, B. Kunert, and W. Stolz, Electron. Lett. 47(16), 931 (2011).

${ }^{17}$ K. Uchida, P. Y. Yu, N. Noto, and E. R. Weber, Appl. Phys. Lett. 64, 2858 (1994).

${ }^{18}$ S. Fuchi, Y. Nonogaki, H. Moriya, A. Koizumi, Y. Fujiwara, and Y. Takeda, Physica E 21, 36 (2004). 
${ }^{19}$ S. Johnson, C. Hong Kuo, M. Boonzaayer, W. Braun, U. Koelle, Y. Zhang, and J. Roth, J. Vac. Sci. Technol. B 16, 1502 (1998).

${ }^{20}$ O. Madelung, Semiconductor Data Handbook, 3rd ed. (Springer, 2004).

${ }^{21}$ J. E. Cunningham, K. W. Goossen, T. H. Chiu, M. D. Williams, W. Jan, and F. Storz, Appl. Phys. Lett. 62, 1236 (1993).

${ }^{22}$ L. G. Wang, P. Kratzer, M. Scheffler, and Q. K. K. Liu, Appl. Phys. A 73, 161 (2001).
${ }^{23}$ I. Vurgaftman and J. R. Meyer, J. Appl. Phys. 89, 5815 (2001).

${ }^{24}$ A. J. Williamson, A. Zunger, and A. Canning, Phys. Rev. B 57, R4253 (1998).

${ }^{25}$ C. Robert, C. Cornet, P. Turban, T. Nguyen Thanh, M. O. Nestoklon, J. Even, J. M. Jancu, M. Perrin, H. Folliot, T. Rohel, S. Tricot, A. Balocchi, D. Lagarde, X. Marie, N. Bertru, O. Durand, and A. Le Corre, Phys. Rev. B 86, 205316 (2012). 